\title{
Mouse model developed for pediatric pituitary hormone deficiency
}

A research team from the USA has successfully developed a mouse model of pediatric pituitary hormone deficiency that separates the endocrine and neural functions of the LHX3 transcription factor.

"Several forms of pediatric pituitary hormone deficiency disease involve mutations in the genes that encode the transcription factors," explains corresponding author Simon Rhodes (Indiana University, Indianapolis, USA). LHX3 has a role in the development of the pituitary gland and nervous system; patients with mutations in LHX3 have pituitary hormone deficiencies and nervous system symptoms.

The researchers identified a novel form of pituitary hormone deficiency (LHX3 Trp224X) in which the patients had deficient expression of pituitary hormones, but had no nervous system problems. They then created a knock-in mouse model of this form of the disease to better understand its etiology. The mice had dwarfism, thyroid disease and female infertility, but did not seem to have nervous system deficits. These results suggest "that the in vivo actions of a transcription factor in different tissues are molecularly separable," says Rhodes.

Mouse models of pediatric pituitary hormone deficiency will enable researchers to carry out experiments to determine the molecular basis of this condition, which would not be possible in human patients. This greater understanding "will allow more precise diagnoses, treatment and genetic counselling for the patients and their families," concludes Rhodes.

\section{Claire Greenhill}

Original article Colvin, S. C. et al. Model of pediatric pituitary hormone deficiency separates the endocrine and neural functions of the LHX3 transcription factor in vivo. Proc. Natl Acad. Sci. USA 108, 173-178 (2011) 